\title{
Research on Control Method of Keeping Flight Formation by Using SDRE on the Sun-Earth Libration Points
}

\author{
He Zhenqi, ${ }^{1,2}$ Zhang Ke, ${ }^{1,2}$ and Lv Meibai ${ }^{1,2}$ \\ ${ }^{1}$ School of Astronautics, Northwestern Polytechnical University, Xi'an, China \\ ${ }^{2}$ National Key Laboratory of Aerospace Flight Dynamics, Xi'an, China \\ Correspondence should be addressed to He Zhenqi; hq774@sohu.com
}

Received 23 October 2016; Revised 30 December 2016; Accepted 17 January 2017; Published 13 February 2017

Academic Editor: Josep M. Trigo-Rodríguez

Copyright (c) 2017 He Zhenqi et al. This is an open access article distributed under the Creative Commons Attribution License, which permits unrestricted use, distribution, and reproduction in any medium, provided the original work is properly cited.

\begin{abstract}
Keeping the flying formation of spacecraft is a key problem which needs to be solved in deep space exploration missions. In this paper, the nonlinear dynamic model of formation flying is established and a series of transformations are carried out on this model equation. By using SDRE (State-Dependent Riccati Equation) algorithm, the optimal control of flying formation is realized. Compared with the traditional control method based on the average orbit elements and LQR (Linear Quadratic Regulator) control method, the SDRE control method has higher control precision and is more suitable for the advantages of continuous control in practical engineering. Finally, the parameter values of the sun-earth libration point L2 are substituted in the equation and simulation is performed. The simulation curves of SDRE controller are compared with LQR controller. The results show that the SDRE controllers time cost is less than the LQR controllers and the former's fuel consumption is less than the latter's in the system transition process.
\end{abstract}

\section{Introduction}

Since the launch of the ISEE-3 mission in 1978, the libration points missions have never stopped. In more than 30 years, a total of 5 spacecrafts have be launched [1]. But all of these are single star missions. Due to the existence of a number of constraints (weight, size, fuel, etc.), the single spacecraft cannot complete the astronomical observation mission with large baseline length or large aperture and high resolution. In recent years, with the rapid development of small spacecraft, the cooperative work of several spacecrafts can complete the formation flying mission, which brings a feasible approach for the astronomical observation mission of high performance and high resolution. At present, it has been highly valued by the international space agency (e.g., NASA, ESA, and research and development institutions in Canada and Japan), and the international space agency put forward a lot of astronomical observation missions, such as XUES, MAXIM, SI, and Darwin [2].

The deployment of spacecraft formation in libration points orbit can be achieved for long baseline interferometry, which is of great significance to the study of the origin of the universe. First of all, the libration points of spacecraft formation includes study the formation configuration design problem, and the problem of formation keeping based on this. Formation keeping is one of the main problems. At present, in the optimal control techniques for formation of libration points, Hamilton and Folta and so forth apply linear optimal control technique to study formation flight control in the vicinity of Lissajous orbit [3]. Penin proposes offline planning and online adjustment algorithm of formation reconfiguration in deep space [4].

SDRE is a nonlinear system control method proposed in the 90s of last century. SDRE uses the same design method as linear system LQR method. It can be regarded as a generalization of LQR control theory in nonlinear systems. Now, some scholars have applied the SDRE method to the design of observers and regulators of low order nonlinear systems, and have achieved good control effects. Weight matrices $Q(x), R(x)$ of SDRE method are the function of system state $x$, but weight matrices $Q, R$ of linear LQR method are real. Therefore, SDRE method is more flexible than LQR method in design and application, the control performance of SDRE method is better than that of LQR method. However, 


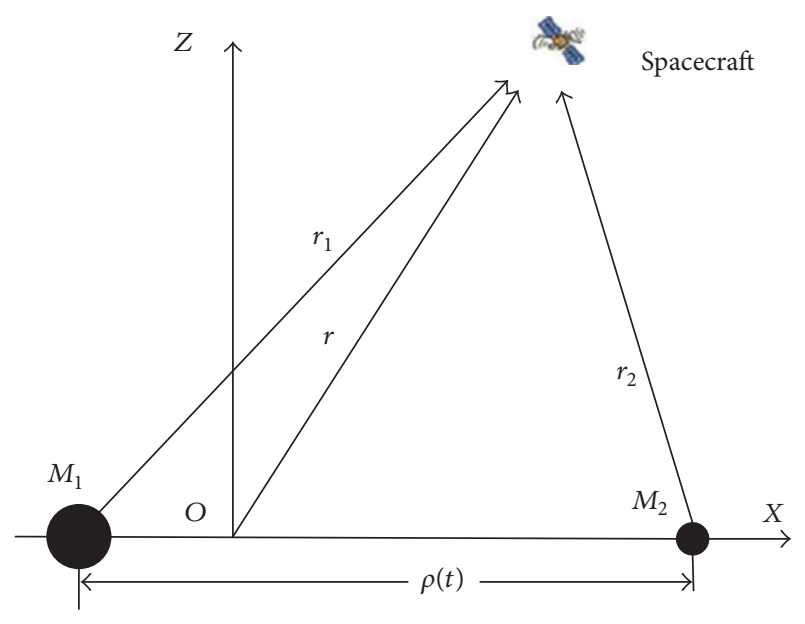

Figure 1: Diagram of $N$-body problem coordinate system.

it will lead to the increase of calculation and unable to get analytical solution, which limits the further application and can only stay at the theoretical level. In recent years, with the development of computer simulation software, human could already get numerical solution and satisfactory results.

In this paper, the nonlinear system motion model is studied by using SDRE nonlinear control method. Theoretically, this method can improve the precision of control and reduce the consumption of fuel. The position error, velocity error, and control quantity of SDRE control method are simulated. The simulation results are compared with LQR control method. Simulation shows that the control accuracy can be improved and the fuel consumption can be reduced.

\section{Establishment of Relative Motion Dynamic Model}

In the coordinate system of $o-x y z$, the coordinates of $M_{1}$ and $M_{2}$ are assumed as $\left[\begin{array}{lll}x_{1} & 0 & 0\end{array}\right]^{T}$ and $\left[\begin{array}{lll}x_{2} & 0 & 0\end{array}\right]^{T}$. The distance between the sun and the earth is $\rho(t)$. The coordinate of the $N$-body is $\left[\begin{array}{lll}x_{N} & y_{N} & z_{N}\end{array}\right]^{T}$. The coordinate of spacecraft is $\left[\begin{array}{lll}x & y & z\end{array}\right]^{T}$. The coordinate system of the $N$-body problem is shown in Figure 1.

The kinetic equation of the spacecraft is

$$
\begin{aligned}
\ddot{x}^{*} & =\left[-\frac{(1-\mu)\left(x^{*}-x_{1}\right)}{r_{1}^{3}}-\frac{\mu\left(x^{*}-x_{2}\right)}{r_{2}^{3}}+\dot{\theta}^{2} x^{*}\right. \\
& \left.+\ddot{\theta} y^{*}+2 \dot{\theta} \dot{y}^{*}\right]+\left[\sum_{n=4}^{N} \frac{\left(-\mu_{N}\left(x^{*}-x_{N}\right)\right)}{r_{N}^{3}}-\frac{\mu_{N} x_{N}}{d_{N}^{3}}\right] \\
& +\left[\widetilde{K} \widetilde{S} \frac{x-x_{s}}{r_{s}^{3}}\right] \\
\ddot{y}^{*} & =\left[-\frac{(1-\mu) y^{*}}{r_{1}^{3}}-\frac{\mu y^{*}}{r_{2}^{3}}+\dot{\theta}^{2} y^{*}+\ddot{\theta} x^{*}+2 \dot{\theta} \dot{x}^{*}\right] \\
& +\left[\sum_{n=4}^{N} \frac{\left(-\mu_{N}\left(y-y_{N}\right)\right)}{r_{N}^{3}}-\frac{\mu_{N} y_{N}}{d_{N}^{3}}\right]+\left[\widetilde{K} \widetilde{S} \frac{y^{*}-y_{s}}{r_{s}^{3}}\right]
\end{aligned}
$$

$$
\begin{aligned}
\ddot{z}^{*} & =\left[-\frac{(1-\mu) z^{*}}{r_{1}^{3}}-\frac{\mu z^{*}}{r_{2}^{3}}\right] \\
& +\left[\sum_{n=4}^{N} \frac{\left(-\mu_{N}\left(z^{*}-z_{N}\right)\right)}{r_{N}^{3}}-\frac{\mu_{N} z_{N}}{d_{N}^{3}}\right]+\left[\widetilde{K} \widetilde{S} \frac{z^{*}-z_{s}}{r_{s}^{3}}\right],
\end{aligned}
$$

where $\left[-(1-\mu)\left(x-x_{1}\right) / r_{1}^{3}-\mu\left(x-x_{2}\right) / r_{2}^{3}+\dot{\theta}^{2} x+\ddot{\theta} y+2 \dot{\theta} \dot{y}\right]$ is the acceleration of the solar gravitational force on the $X$ axis of the main spacecraft; $\left[\sum_{n=4}^{N}\left(\left(-\mu_{N}\left(y-y_{N}\right)\right) / r_{N}^{3}\right)-\mu_{N} y_{N} / d_{N}^{3}\right]$ is the perturbation acceleration of the $N$-body spacecraft on the $X$ axis; $\left[\widetilde{K} \widetilde{S}\left(\left(x-x_{s}\right) / r_{s}^{3}\right)\right]$ is the perturbation acceleration of solar radiation on spacecraft $x$-axis; the $y$-axis and $z$-axis are the same as the $X$ axis. Among them, $\theta$ is the initial phase angle; $\mu_{N}$ represents the normalized quality of the $N$-body; $\widetilde{K}$ is the nondimensional solar reflection constant; $\widetilde{S}$ is the solar flux. Then

$$
\begin{aligned}
& r_{1}=\left[\left(x^{*}-x_{1}\right)^{2}+\left(y^{*}\right)^{2}+\left(z^{*}\right)^{2}\right]^{1 / 2} \\
& r_{2}=\left[\left(x^{*}-x_{2}\right)^{2}+\left(y^{*}\right)^{2}+\left(z^{*}\right)^{2}\right]^{1 / 2} \\
& r_{N}=\left[\left(x^{*}-x_{N}\right)^{2}+\left(y^{*}-y_{N}\right)^{2}+\left(z^{*}-z_{N}\right)^{2}\right]^{1 / 2} \\
& d_{N}=\left[x_{N}^{2}+y_{N}^{2}+z_{N}^{2}\right]^{1 / 2} \\
& r_{s}=\left[\left(x^{*}-x_{s}\right)^{2}+\left(y^{*}-y_{s}\right)^{2}+\left(z^{*}-z_{s}\right)^{2}\right]^{1 / 2} .
\end{aligned}
$$

Assuming that the main spacecraft is $S^{A}$, the slave spacecraft is $S^{B}$; their position vectors are $r^{A}=\left[\begin{array}{lll}x_{A} & y_{A} & z_{A}\end{array}\right]^{T}$ and $r^{B}=\left[\begin{array}{lll}x_{B} & y_{B} & z_{B}\end{array}\right]^{T}$; the position vector of the slave spacecraft is $r=\left[\begin{array}{lll}x & y & z\end{array}\right]^{T} \cdot r_{1}^{A}$ and $r_{2}^{A}$ represent the distance vector between $M_{1}$ and $M_{2}$ to $S^{A} ; r_{1}^{B}$ and $r_{2}^{B}$ represent the distance vector between $M_{1}$ and $M_{2}$ to $S^{B}$. Libration point orbit spacecraft relative motion diagram is shown in Figure 2.

The nonlinear model of relative motion of the spacecraft is

$$
\ddot{r}=\ddot{r}^{B}-\ddot{r}^{A}
$$

According to (1), the sum of the perturbation force and control force can be expressed as $f$. The nonlinear relative motion equation of $S^{B}$ to $S^{A}$ can be obtained:

$$
\begin{aligned}
\ddot{x}= & -\frac{(1-\mu)\left(x^{A}+x-x_{1}\right) \sigma_{1}+x}{\left(r_{1}^{A}\right)^{3}} \\
& -\frac{\mu\left(x^{A}+x-x_{2}\right) \sigma_{2}+x}{\left(r_{2}^{A}\right)^{3}}+\dot{\theta}^{2} x+\ddot{\theta} y+2 \dot{\theta} \dot{y} \\
& +f_{x} \\
\ddot{y}= & -\frac{(1-\mu)\left(y^{A}+y\right) \sigma_{1}+y}{\left(r_{1}^{A}\right)^{3}}-\frac{\mu\left(y^{A}+y\right) \sigma_{2}+y}{\left(r_{2}^{A}\right)^{3}} \\
& +\dot{\theta}^{2} y-\ddot{\theta} x-2 \dot{\theta} \dot{x}+f_{y}
\end{aligned}
$$




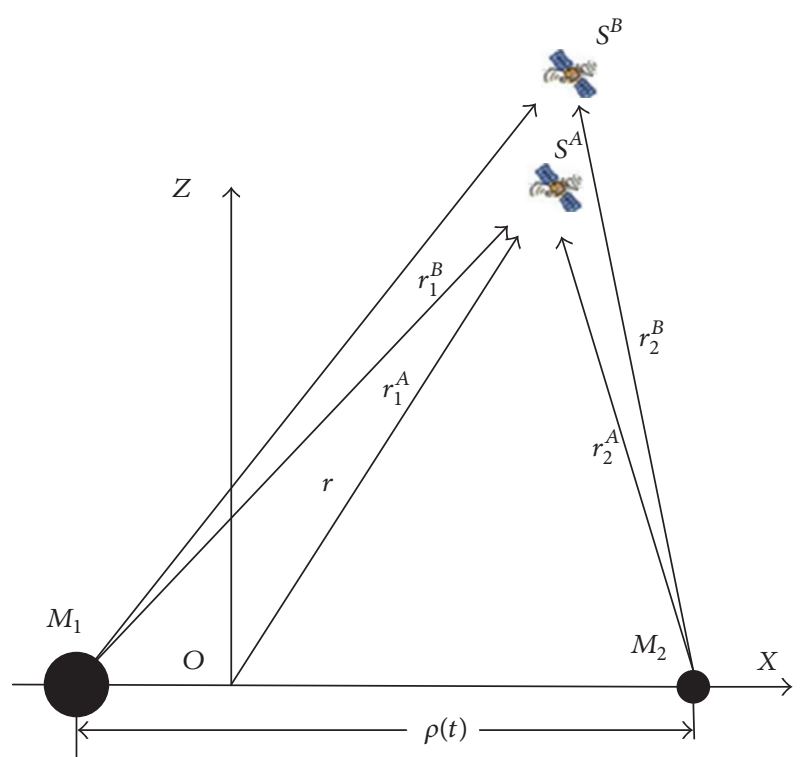

Figure 2: Diagram of libration point orbit spacecraft relative motion.

$$
\begin{aligned}
\ddot{z}= & -\frac{(1-\mu)\left(z^{A}+z\right) \sigma_{1}+z}{\left(r_{1}^{A}\right)^{3}}-\frac{\mu\left(z^{A}+z\right) \sigma_{2}+z}{\left(r_{2}^{A}\right)^{3}} \\
& +f_{z},
\end{aligned}
$$

where $f_{x}, f_{y}$, and $f_{z}$ are the three components of the sum of perturbation force and control force. $r_{1}^{A}, r_{2}^{A}$ represent the distance vector between $M_{1}$ and $M_{2}$ to $S^{A}$, and $r_{1}^{A}, r_{2}^{A}$ expressions are

$$
\begin{aligned}
& r_{1}^{A}=\left[\left(x^{A}-x_{1}\right)^{2}+\left(y^{A}\right)^{2}+\left(z^{A}\right)^{2}\right]^{1 / 2} \\
& r_{2}^{A}=\left[\left(x^{A}-x_{2}\right)^{2}+\left(y^{A}\right)^{2}+\left(z^{A}\right)^{2}\right]^{1 / 2} .
\end{aligned}
$$

$r_{1}^{B}, r_{2}^{B}$ represent the distance vector between $M_{1}$ and $M_{2}$ to $S^{B}$, and $r_{1}^{B}, r_{2}^{B}$ expressions are

$$
\begin{aligned}
& r_{1}^{B}=\left[\left(x^{A}+x-x_{1}\right)^{2}+\left(y^{A}+y\right)^{2}+\left(z^{A}+z^{2}\right)\right]^{1 / 2} \\
& r_{2}^{B}=\left[\left(x^{A}+x-x_{2}\right)^{2}+\left(y^{A}+y\right)^{2}+\left(z^{A}+z^{2}\right)\right]^{1 / 2}
\end{aligned}
$$

where $\sigma_{1}$ and $\sigma_{2}$ expressions are

$$
\begin{aligned}
& \sigma_{1}=\left(\frac{r_{1}^{A}}{r_{1}^{B}}\right)^{3}-1 \\
& \sigma_{2}=\left(\frac{r_{2}^{A}}{r_{2}^{B}}\right)^{3}-1 .
\end{aligned}
$$

In (4), if $\dot{\theta}=1, x_{1}=-\mu$, and $x_{2}=1-\mu$ can get dynamic equation of circular restricted libration point orbit (Hill orbit) nonlinear relative motion:

$$
\begin{aligned}
\ddot{x}= & -\frac{(1-\mu)\left(x^{A}+x-x_{1}\right) \sigma_{1}+x}{\left(r_{1}^{A}\right)^{3}} \\
& -\frac{\mu\left(x^{A}+x-x_{2}\right) \sigma_{2}+x}{\left(r_{2}^{A}\right)^{3}}+x+2 \dot{y}+f_{x} \\
\ddot{y}= & -\frac{(1-\mu)\left(y^{A}+y\right) \sigma_{1}+y}{\left(r_{1}^{A}\right)^{3}}-\frac{\mu\left(y^{A}+y\right) \sigma_{2}+y}{\left(r_{2}^{A}\right)^{3}} \\
& +y-2 \dot{x}+f_{y} \\
\ddot{z}= & -\frac{(1-\mu)\left(z^{A}+z\right) \sigma_{1}+z}{\left(r_{1}^{A}\right)^{3}}-\frac{\mu\left(z^{A}+z\right) \sigma_{2}+z}{\left(r_{2}^{A}\right)^{3}} \\
& +f_{z} .
\end{aligned}
$$

Formula (8) carries out a series of simple transformations and removes the constant term:

$$
\begin{aligned}
\ddot{x}= & -\frac{(1-\mu)\left(x^{A}+\mu\right)}{\left(r_{1}^{B}\right)^{3}}-\frac{(1-\mu)}{\left(r_{1}^{B}\right)^{3}} x-\frac{\mu\left(x^{A}-1+\mu\right)}{\left(r_{2}^{B}\right)^{3}} \\
& -\frac{\mu}{\left(r_{2}^{B}\right)^{3}} x+x+2 \dot{y}+f_{x} \\
\ddot{y}= & -\frac{(1-\mu) y^{A}}{\left(r_{1}^{B}\right)^{3}}-\frac{(1-\mu)}{\left(r_{1}^{B}\right)^{3}} y-\mu \frac{\left(y^{A}\right)}{\left(r_{2}^{B}\right)^{3}}-\frac{\mu}{\left(r_{2}^{B}\right)^{3}} y+y \\
& -2 \dot{x}+f_{y} \\
\ddot{z}= & -\frac{(1-\mu) z^{A}}{\left(r_{1}^{B}\right)^{3}}-\frac{1-\mu}{\left(r_{1}^{B}\right)^{3}} z-\mu \frac{\left(z^{A}\right)}{\left(r_{2}^{B}\right)^{3}}-\frac{\mu}{\left(r_{2}^{B}\right)^{3}} z+f_{z} .
\end{aligned}
$$

\section{Establishment of SDC Model}

When using the SDRE method, the nonlinear system dynamics equations are transformed into SDC (state-dependent coefficients) form $[5,6]$.

Nonlinear affine system:

$$
\dot{x}=f(x)+g(x) u
$$

SDC forms can be obtained by pseudolinearization of nonlinear affine systems [7]:

$$
\dot{x}=A(x) x+B(x) u
$$

Formula (9) can be converted into

$$
\begin{aligned}
\ddot{x}= & \left(x^{A}+\mu\right)\left[\frac{(\mu-1)}{\left(r_{1}^{B}\right)^{3}}-\frac{\mu}{\left(r_{2}^{B}\right)^{3}}\right]-\frac{\mu}{\left(r_{2}^{B}\right)^{3}} \\
& +\left[\frac{(\mu-1)}{\left(r_{1}^{B}\right)^{3}}-\frac{\mu}{\left(r_{2}^{B}\right)^{3}}\right] x+x+2 \dot{y}+f_{x}
\end{aligned}
$$


4

Advances in Astronomy

$$
\begin{aligned}
\ddot{y}= & y^{A}\left[\frac{(\mu-1)}{\left(r_{1}^{B}\right)^{3}}-\frac{\mu}{\left(r_{2}^{B}\right)^{3}}\right]-\left[\frac{(\mu-1)}{\left(r_{1}^{B}\right)^{3}}-\frac{\mu}{\left(r_{2}^{B}\right)^{3}}\right] y \\
& +y-2 \dot{x}+f_{y} \\
\ddot{z}= & z^{A}\left[\frac{(\mu-1)}{\left(r_{1}^{B}\right)^{3}}-\frac{\mu}{\left(r_{2}^{B}\right)^{3}}\right]-\left[\frac{(\mu-1)}{\left(r_{1}^{B}\right)^{3}}-\frac{\mu}{\left(r_{2}^{B}\right)^{3}}\right] z \\
& +f_{z} .
\end{aligned}
$$

Assume that all states of the system can be measured, known as full state feedback SDRE implementation, where

$$
\begin{aligned}
& \frac{(\mu-1)}{\left(r_{1}^{B}\right)^{3}}-\frac{\mu}{\left(r_{2}^{B}\right)^{3}}=(\mu-1)\left[\left(x^{A}+x-x_{1}\right)^{2}\right. \\
& \left.+\left(y^{A}+y\right)^{2}+\left(z^{A}+z\right)^{2}\right]^{-1 / 2}-\mu\left[\left(x^{A}+x-x^{2}\right)^{2}\right. \\
& \left.+\left(y^{A}+y\right)^{2}+\left(z^{A}+z\right)^{2}\right]^{-1 / 2}=(\mu-1) \\
& +\left[\left(x^{A}-x_{1}\right)^{2}+\left(y^{A}\right)^{2}+\left(z^{A}\right)^{2}+x^{2}+y^{2}+z^{2}\right. \\
& \left.+2\left(x^{A}-x_{1}\right) x+2 y^{A} y+2 z^{A} z\right]^{-3 / 2} \\
& -\mu\left[\left(x^{A}-x_{2}\right)^{2}+\left(y^{A}\right)^{2}+\left(z^{A}\right)^{2}+x^{2}+y^{2}+z^{2}\right. \\
& \left.+2\left(x^{A}-x_{2}\right) x+2 y^{A} y+2 z^{A} z\right]^{-3 / 2} .
\end{aligned}
$$

$$
\begin{aligned}
& \psi=1+\psi_{1}+\psi_{2}+\cdots+\psi_{n}+\cdots ; \quad \psi_{1}=-\frac{3}{2} \frac{\xi_{1}}{a}, \psi_{2}=-\frac{5}{4} \psi_{1} \frac{\xi_{1}}{a}, \psi_{3}=-\frac{7}{6} \psi_{2} \frac{\xi_{1}}{a}, \ldots, \psi_{n}=-\frac{2 n+1}{2 n} \psi_{n-1} \frac{\xi_{1}}{a}, \ldots \\
& \varphi=1+\varphi_{1}+\varphi_{2}+\cdots+\varphi_{n}+\cdots ; \quad \varphi_{1}=-\frac{3}{2} \frac{\xi_{2}}{b}, \varphi_{2}=-\frac{5}{4} \varphi_{1} \frac{\xi_{2}}{b}, \varphi_{3}=-\frac{7}{6} \varphi_{2} \frac{\xi_{2}}{b}, \ldots, \varphi_{n}=-\frac{2 n+1}{2 n} \varphi_{n-1} \frac{\xi_{2}}{b}, \ldots
\end{aligned}
$$

$$
\begin{aligned}
\xi_{2}= & {\left[2\left(x^{A}-x_{2}\right)+x\right] x+\left(2 y^{A}+y\right) y } \\
& +\left(2 z^{A}+z\right) z .
\end{aligned}
$$

Application of Taylor series:

Similarly

$$
\begin{aligned}
& \frac{(\mu-1)}{\left(r_{1}^{B}\right)^{3}}-\frac{\mu}{\left(r_{2}^{B}\right)^{3}}=(\mu-1)\left[\left(x^{A}-x_{1}\right)^{2}+\left(y^{A}\right)^{2}\right. \\
& \left.+\left(z^{A}\right)^{2}+x^{2}+y^{2}+z^{2}+\xi_{1}\right]^{-3 / 2}-\mu\left[\left(x^{A}-x_{2}\right)^{2}\right. \\
& \left.+\left(y^{A}\right)^{2}+\left(z^{A}\right)^{2}+\xi_{2}\right]^{-3 / 2}=(\mu-1) a^{-3 / 2} \\
& -\mu b^{-3 / 2}+\mu b^{-5 / 2} \frac{3}{2} \varphi\left[2\left(x^{A}-x_{2}\right)+x\right]-(\mu-1) \\
& \cdot a^{-5 / 2} \frac{3}{2} \psi\left[2\left(x^{A}-x_{1}\right)+x\right] x+\left[\mu b^{-5 / 2} \frac{3}{2} \varphi\right. \\
& \left.-(\mu-1) a^{-5 / 2} \frac{3}{2} \psi\right]\left(2 y^{A}+y\right) y+\left[\mu b^{-5 / 2} \frac{3}{2} \varphi\right. \\
& \left.-(\mu-1) a^{-5 / 2} \frac{3}{2} \psi\right]\left(2 z^{A}+z\right) z .
\end{aligned}
$$

$$
\begin{aligned}
\frac{\mu}{\left(r_{2}^{B}\right)^{3}}= & \mu b^{-5 / 2} \frac{3}{2} \varphi\left[2\left(x^{A}-x_{2}\right)+x\right] x+\left(2 y^{A}+y\right) y \\
& +\left(2 z^{A}+z\right) z,
\end{aligned}
$$

Formula (12) can be further written as

$$
\begin{aligned}
\ddot{x}= & \left(x^{A}+\mu\right) \mu b^{-5 / 2} \frac{3}{2} \varphi\left[2\left(x^{A}-x_{2}\right)+x\right]-(1-\mu) \\
& \cdot a^{-5 / 2} \frac{3}{2} \psi\left[2\left(x^{A}-x_{1}\right)+x\right] x-\mu b^{-5 / 2} \frac{3}{2} \\
& \cdot \varphi\left[2\left(x^{A}-x_{2}\right)+x\right] x+\left[\frac{\mu-1}{\left(r_{1}^{B}\right)^{3}}-\frac{\mu}{\left(r_{2}^{B}\right)^{3}}\right] x+x \\
& +\left(x^{A}+\mu\right)\left[\mu b^{-5 / 2} \frac{3}{2} \varphi-(\mu-1) a^{-5 / 2} \frac{3}{2} \psi\right]
\end{aligned}
$$


Advances in Astronomy

5

$$
\begin{aligned}
& +y^{A}\left[\mu b^{-5 / 2} \frac{3}{2} \varphi-(\mu-1) a^{-5 / 2} \frac{3}{2} \psi\right]\left(2 y^{A}+y\right) y \\
& -\left[\frac{\mu-1}{\left(r_{1}^{B}\right)^{3}}-\frac{\mu}{\left(r_{2}^{B}\right)^{3}}\right] y+y \\
& +y^{A}\left[\mu b^{-5 / 2} \frac{3}{2} \varphi-(\mu-1) a^{-5 / 2} \frac{3}{2} \psi\right]\left(2 z^{A}+z\right) z \\
& -\mu b^{-5 / 2} \frac{3}{2} \varphi\left(2 z^{A}+z\right) z+2 \dot{x}+f_{y} \\
& \ddot{z}=z^{A} \mu b^{-5 / 2} \frac{3}{2} \varphi\left[2\left(x^{A}-x_{2}\right)+x\right]-(1-\mu) a^{-5 / 2} \frac{3}{2} \\
& \cdot \psi\left[2\left(x^{A}-x_{1}\right)+x\right] x \\
& +z^{A}\left[\mu b^{-5 / 2} \frac{3}{2} \varphi-(\mu-1) a^{-5 / 2} \frac{3}{2} \psi\right]\left(2 y^{A}+y\right) y \\
& -\left[\frac{\mu-1}{\left(r_{1}^{B}\right)^{3}}-\frac{\mu}{\left(r_{2}^{B}\right)^{3}}\right] y \\
& +z^{A}\left[\mu b^{-5 / 2} \frac{3}{2} \varphi-(\mu-1) a^{-5 / 2} \frac{3}{2} \psi\right]\left(2 z^{A}+z\right) z \\
& -\mu b^{-5 / 2} \frac{3}{2} \varphi\left(2 z^{A}+z\right) z+2 \dot{x}+f_{z} \text {. }
\end{aligned}
$$

4. Design of SDRE Controller

Suppose

$$
\begin{aligned}
x= & {\left[\begin{array}{llllll}
x_{1} & x_{2} & x_{3} & x_{4} & x_{5} & x_{6}
\end{array}\right]^{T} } \\
= & {\left[\begin{array}{llllll}
x & y & z & \dot{x} & \dot{y} & \dot{z}
\end{array}\right]^{T} } \\
u & =\left[\begin{array}{lll}
u_{1} & u_{2} & u_{3}
\end{array}\right]^{T} \\
{\left[\begin{array}{c}
\dot{x}_{1} \\
\dot{y}_{2} \\
\dot{z}_{3} \\
\dot{x}_{4} \\
\dot{y}_{5} \\
\dot{z}_{6}
\end{array}\right]=} & {\left[\begin{array}{cccccc}
0 & 0 & 0 & 1 & 0 & 0 \\
0 & 0 & 0 & 0 & 1 & 0 \\
0 & 0 & 0 & 0 & 0 & 1 \\
a_{41} & a_{42} & a_{43} & a_{44} & a_{45} & a_{46} \\
a_{51} & a_{52} & a_{53} & a_{54} & a_{55} & a_{56} \\
a_{61} & a_{62} & a_{63} & a_{64} & a_{65} & a_{66}
\end{array}\right]\left[\begin{array}{l}
x_{1} \\
x_{2} \\
x_{3} \\
x_{4} \\
x_{5} \\
x_{6}
\end{array}\right] } \\
& +\left[\begin{array}{lll}
0 & 0 & 0 \\
0 & 0 & 0 \\
0 & 0 & 0 \\
1 & 0 & 0 \\
0 & 1 & 0 \\
0 & 0 & 1
\end{array}\right]\left[\begin{array}{l}
u_{1} \\
u_{2} \\
u_{3}
\end{array}\right],
\end{aligned}
$$

(19) 


$$
\begin{aligned}
a_{63} & =z^{A}\left[\mu b^{-5 / 2} \frac{3}{2} \varphi-(\mu-1) a^{-5 / 2} \frac{3}{2} \psi\right]\left(2 z^{A}+z\right) \\
& -\mu b^{-5 / 2} \frac{3}{2} \varphi\left(2 z^{A}+z\right) \\
a_{64} & =0
\end{aligned}
$$

$a_{65}=0$

$a_{66}=0$.

System controllability matrix:

$$
W_{c}=\left[\begin{array}{llllll}
A(x) & A(x) B(x) & A^{2}(x) B(x) & A^{3}(x) B(x) & A^{4}(x) B(x) & A^{5}(x) B(x)
\end{array}\right] .
$$

The calculation shows that the system is controlled point by point.

And the objective function of SDRE theory is

$$
\begin{aligned}
& J_{c} \\
& \quad=\frac{1}{2} \int_{t_{0}}^{\infty}\left[\left(x-x_{d}\right)^{T} Q(x)\left(x-x_{d}\right)+u^{T} R(x) u\right] d t .
\end{aligned}
$$

In the equation, $x_{d}$ is an ideal state, $Q(x)$ is used as the weight matrix of the error in the optimization process, and $R(x)$ is used as the weight matrix of the control variables in the optimization process. They are functions of the system state $x$.

From (11), because the solution of $x$ is not unique and $A(x)$ is a function of $x$, the choice of $A(x)$ is not unique. Papers $[8,9]$ will lead to suboptimal solution. Solving Riccati equation

$$
\begin{aligned}
& P(x) A(x)+A^{T}(x) P(x)-P(x) B(x) R^{-1}(x) P(x) \\
& \quad+Q(x)
\end{aligned}
$$

$P(x)>0$ can be solved.

The control law of the nonlinear system in the performance index is

$$
u=-R^{-1}(x) B(x) P(x) x .
$$

\section{Numerical Example and Simulation Results}

Table 1 shows the corresponding basic constants of the L2 point of the sun-earth system. According to these parameters we can obtain simulation curves through control method of keeping flight formation by using SDRE on the sun-earth libration points.

Under ideal conditions, the initial relative position and velocity of the leader-satellite and the following-satellite formations are

$$
\begin{aligned}
& x_{d}=-20 \mathrm{~m}, \\
& y_{d}=160 \mathrm{~m}, \\
& z_{d}=0 \mathrm{~m}, \\
& \dot{x}_{d}=0.1 \mathrm{~m} / s, \\
& \dot{y}_{d}=-0.1 \mathrm{~m} / \mathrm{s}, \\
& \dot{z}_{d}=0 \mathrm{~m} / s .
\end{aligned}
$$

Under disturbance, the initial relative position and velocity error are

$$
\begin{aligned}
& x=30 \mathrm{~m}, \\
& y=180 \mathrm{~m}, \\
& z=-10 \mathrm{~m}, \\
& \dot{x}=0.4 \mathrm{~m} / \mathrm{s}, \\
& \dot{y}=-0.2 \mathrm{~m} / \mathrm{s}, \\
& \dot{z}=0.1 \mathrm{~m} / \mathrm{s} .
\end{aligned}
$$

So the initial relative position error and velocity error between the leader-satellite and the following-satellite are

$$
x-x_{d}=\left[\begin{array}{llllll}
50 & 20 & -10 & 0.2 & 0.3 & 0.1
\end{array}\right] .
$$

Without the influence of the perturbation, in order to compare the effect of LQR controller and SDRE controller, in the LQR controller, the weight matrices $Q(x)$ and $R(x)$ are, respectively,

$$
\begin{aligned}
& Q(x)=\operatorname{diag}\left(\left[x_{1}^{2}, x_{3},\left(\dot{x}_{3}+x_{1}\right)^{3}, x_{2}, \dot{x}_{1}, x_{1}\right]\right) ; \\
& R(x) \\
& \quad=\operatorname{diag}\left(\left[100+x_{1}+x_{2}, 100+x_{2}-x_{3}, 300+\dot{x}_{1}^{2}\right]\right) .
\end{aligned}
$$

Running under the MATLAB to get the simulation results of various states are shown in Figures 3-5.

The nonlinear terms in the nonlinear equation (12) are deleted. Then, the linear equation can be obtained:

$$
\begin{aligned}
& \ddot{x}=\left[\frac{(\mu-1)}{\left(r_{1}^{B^{*}}\right)^{3}}-\frac{\mu}{\left(r_{2}^{B^{*}}\right)^{3}}+1\right] x+2 \dot{y}+f_{x} \\
& \ddot{y}=\left[\frac{(\mu-1)}{\left(r_{1}^{B^{*}}\right)^{3}}-\frac{\mu}{\left(r_{2}^{B^{*}}\right)^{3}}+1\right] y-2 \dot{x}+f_{y} \\
& \ddot{z}=\left[\frac{(\mu-1)}{\left(r_{1}^{B^{*}}\right)^{3}}-\frac{\mu}{\left(r_{2}^{B^{*}}\right)^{3}}\right] z+f_{z} .
\end{aligned}
$$

It becomes a linear equation. Afterwards the LQR control method is used to perform simulation. In order to compare the effect of the controller designed by LQR method and 
TABLE 1: Basic constants of L2 point in the sun-earth system.

\begin{tabular}{lc}
\hline Class (shorthand) & Value \\
\hline Solar mass $\left(M_{1}\right)$ & $1.989 \times 10^{30} \mathrm{~kg}$ \\
Earth mass + lunar mass $\left(M_{2}\right)$ & $5.976 \times 10^{24} \mathrm{~kg}+7.196 \times 10^{22} \mathrm{~kg}$ \\
Distance from the $O$ point to the sun $\left(D_{1}\right)$ & $4.9027779 \times 10^{3} \mathrm{~km}$ \\
Distance from the $O$ point to the earth - moon system $\left(D_{2}\right)$ & $4.5484085 \times 10^{8} \mathrm{~km}$ \\
The orbital eccentricity $(e)$ & 0.01670862 \\
Distance from the $O$ point to the L2 libration point $\left(x_{e}\right)$ & $1.5110515 \times 10^{8} \mathrm{~km}$ \\
\hline
\end{tabular}

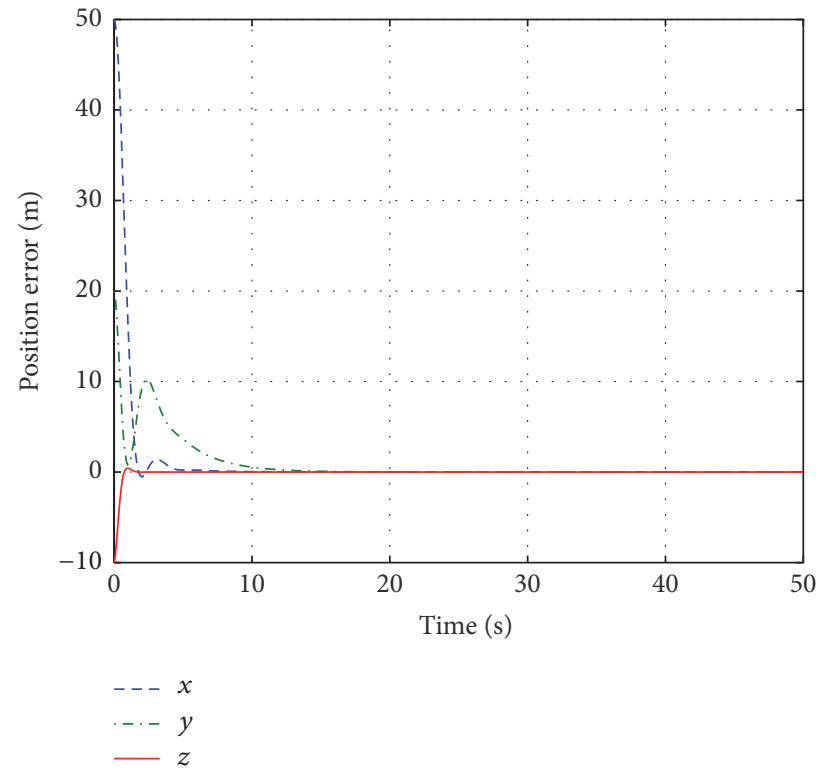

FIGURE 3: Position error variation curve of SDRE.

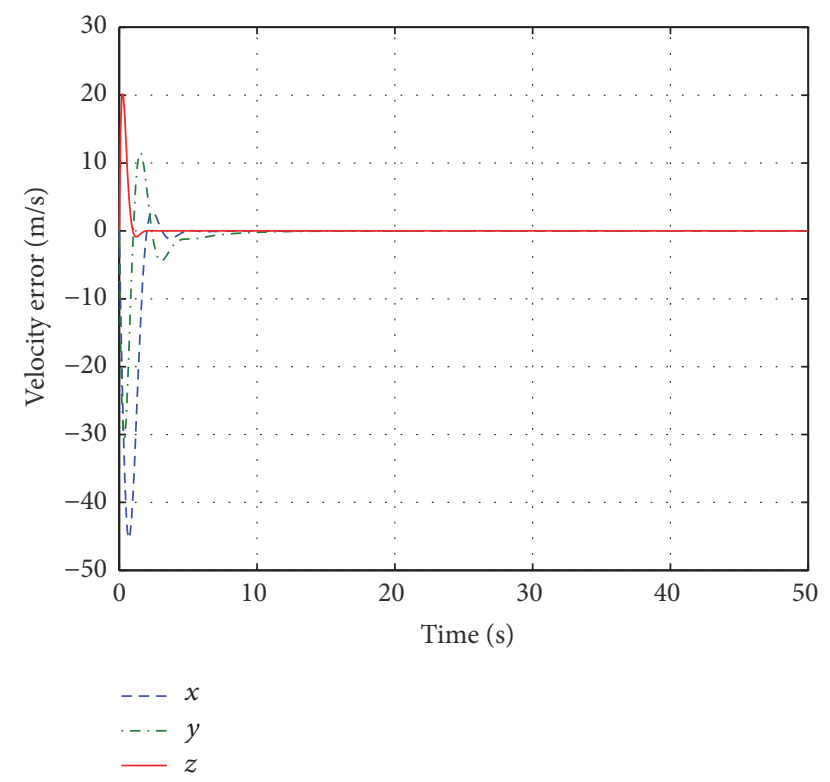

FIGURE 4: Velocity error variation curve of SDRE.

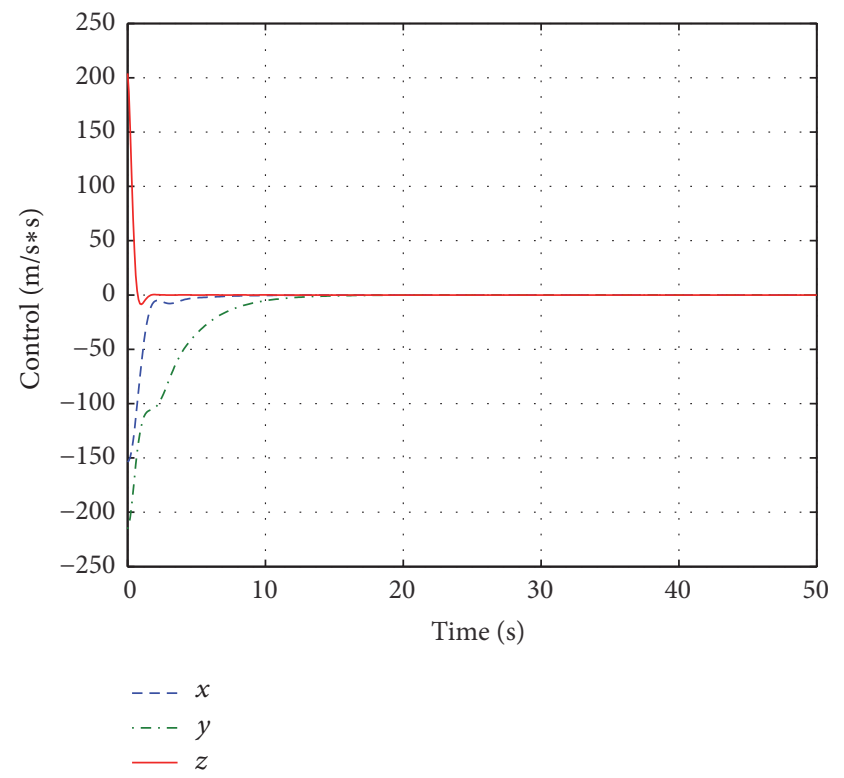

FIGURE 5: Control volume change curve of SDRE.

SDRE method, we take the following weight matrices $Q$ and $R$ in the LQR control method:

$$
\begin{aligned}
& Q=\operatorname{diag}([1,1,1,1,1,1]) ; \\
& R=\operatorname{diag}([300,300,300]) .
\end{aligned}
$$

It is advisable to take the following weight matrices $Q(x)$ and $R(x)$ in the SDRE control method:

$$
\begin{aligned}
& Q(x)=\operatorname{diag}\left(\left[1+\left|x_{1}\right|+\left|x_{2}\right|, 1+\left|x_{2}\right|, 1+\left|x_{3}\right|, 1\right.\right. \\
& \left.\left.\quad+\left|\dot{x}_{1}\right|, 1+\left|\dot{x}_{2}\right|, 1+\left|\dot{x}_{3}\right|\right]\right) ; \\
& R(x)=\operatorname{diag}\left(\left[300+x_{1}^{3}, 300+x_{2}^{4}, 300+x_{3}^{4}\right]\right) .
\end{aligned}
$$

The simulation results of various states are shown in Figures 6-14.

In the LQR method, the choice of the weight matrices $Q$ and $R$ has a great influence on the performance of the controller. With the increase of the $Q$ value, the position and velocity error curve will converge quickly, and the working time of the orbit control engine will be reduced. At the same time, the amplitude of the velocity error and the control 


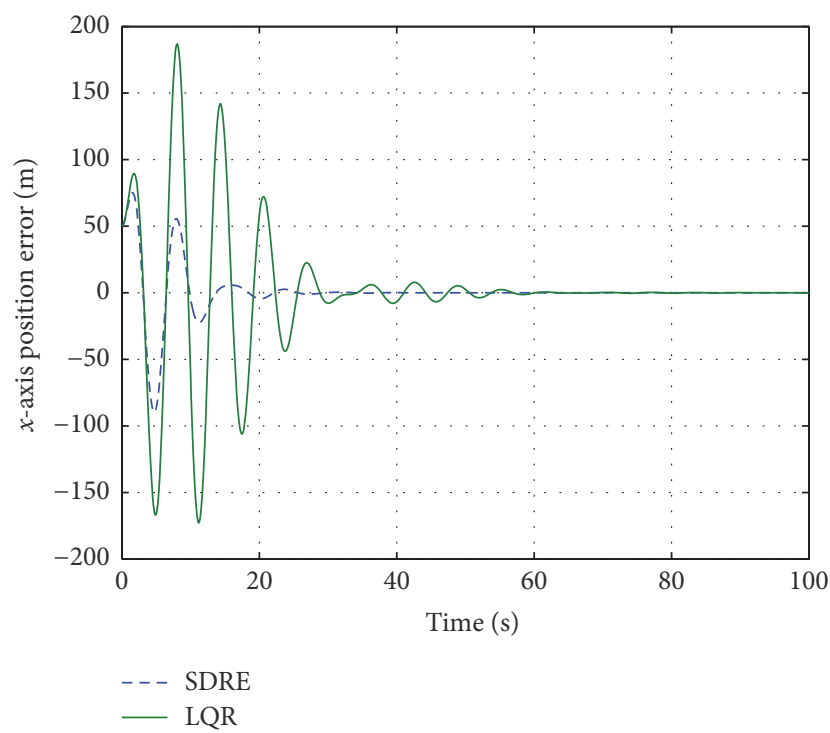

FIgURE 6: $X$ axis position error variation curve.

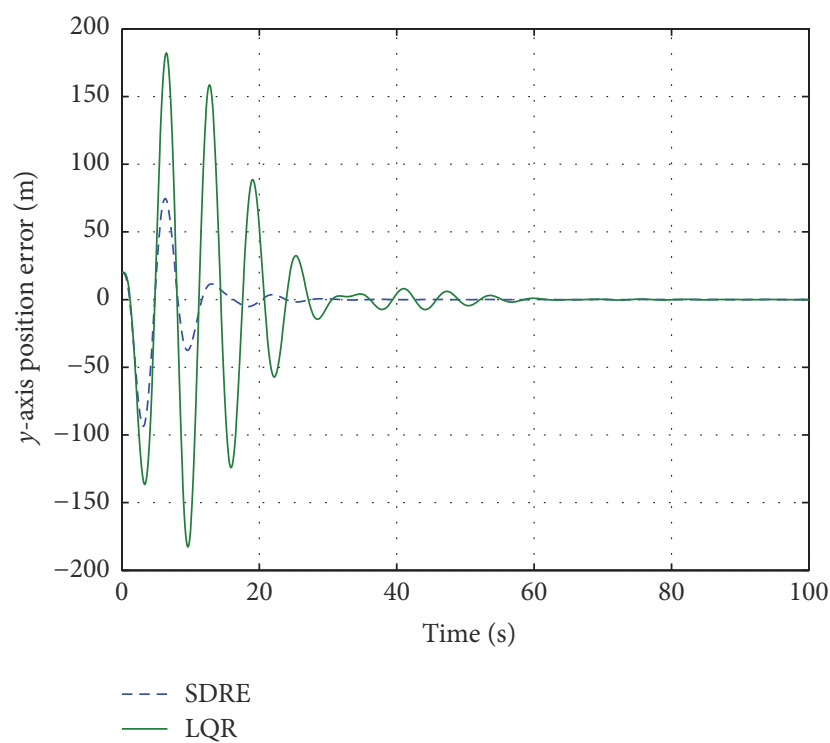

FIGURE 7: $Y$ axis position error variation curve.

quantity will increase. But, with the increase of the value of $R$, they show the opposite situation. So, it is very difficult to choose the weight matrices $Q$ and $R$. In practice, the method of cut-and-trial is generally adopted. It depends on the designer's experience.

In the SDRE method, the weight matrices $Q(x)$ and $R(x)$ are a function of state $x$, which is more effective for state control. The choice of $Q(x)$ and $R(x)$ is more flexible. But the effect of SDRE method on position error curve, velocity error curve and control curve is the same as that of LQR method. In practice, the method of cut-and-trial is also generally adopted.

In Figures 3-5, the weight matrices $Q$ and $R$ are all taken as a function of the state $x$. As is seen from the simulation curve, the whole convergence process is short and smooth.

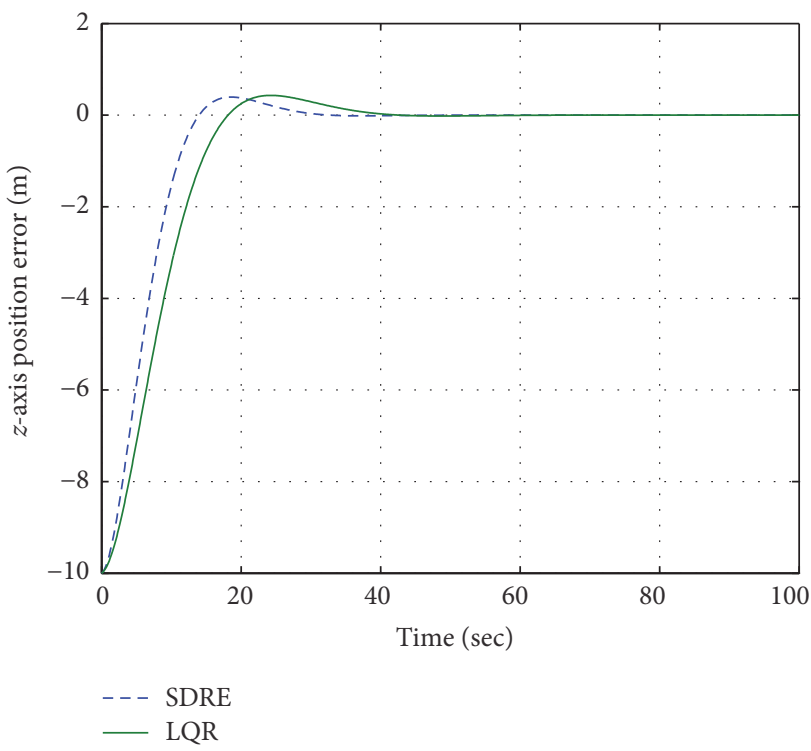

FIGURE 8: $Z$ axis position error variation curve.

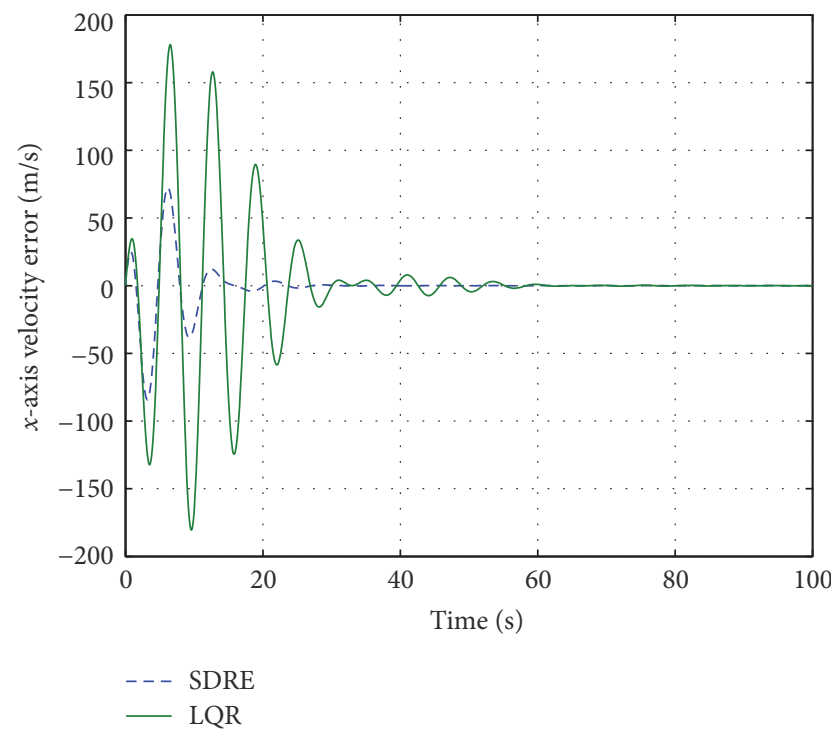

FIGURE 9: $X$ axis velocity error variation curve.

In Figures 6 and 7, these are position error curves of $X$ axis and $Y$ axis. The overshooting volume of the SDRE method is obviously less than that of the LQR method, and the time to reach the steady state of the SDRE method is also significantly less than that of the LQR method. In Figure 8, The overshooting volume of the SDRE method and the LQR method are almost the same. But the time to reach the steady state of SDRE method is significantly less than that of the LQR method. In Figures 9 and 10, these are velocity error curves of $X$ axis and $Y$ axis, the overshooting volume of the SDRE method is obviously less than that of the LQR method, and the time to reach the steady state is also significantly less than that of LQR method. In Figure 11, the overshooting volume of the SDRE method is slightly larger than that of the LQR method, but the time of reaching steady state of the 


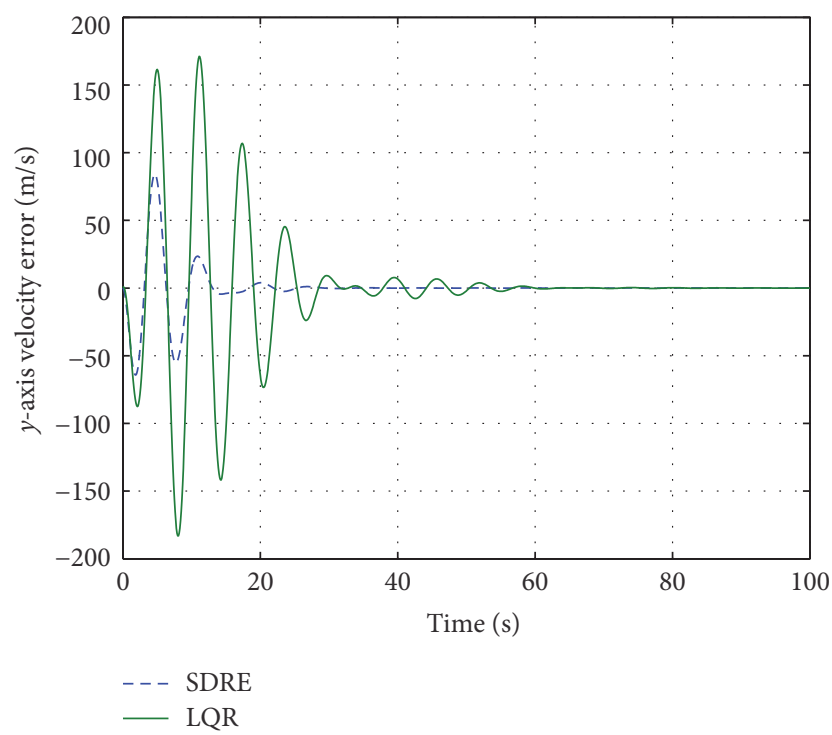

FIgURE 10: $Y$ axis velocity error variation curve.

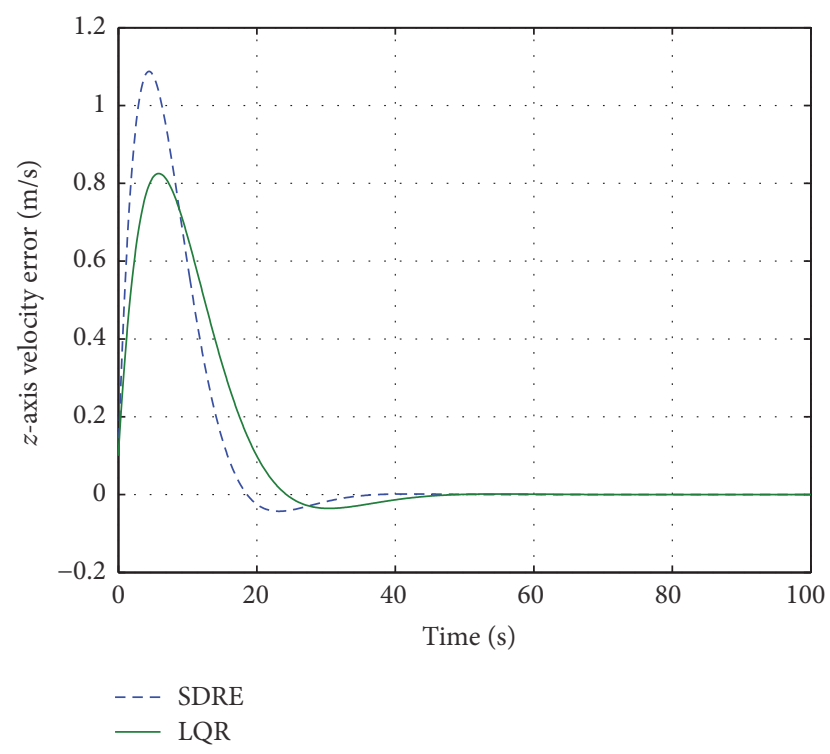

FIGURE 11: $Z$ axis velocity error variation curve.

SDRE method is less than that of LQR method. In Figures 12 and 13, the overshooting volume of the SDRE method is less than that of the LQR method, but the control time is almost the same. In Figure 14, although the overshooting volume of the SDRE method is slightly larger, the control time is much shorter. Therefore, the SDRE control method is superior to the LQR control method on control force. And the fuel consumption of the SDRE method is much less than that of the LQR method in the case of steady state.

\section{Conclusions}

In the simulation diagram the larger overshooting volume, the shorter time to reach steady state; the smaller overshooting volume, the longer time to steady state. In the LQR control

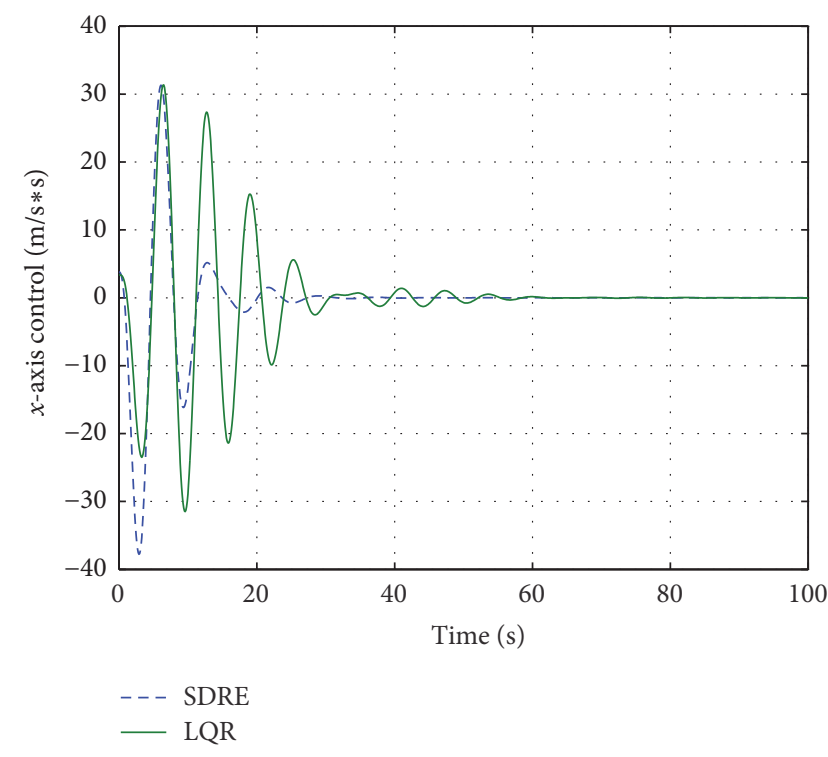

Figure 12: $X$ axis control change curve.

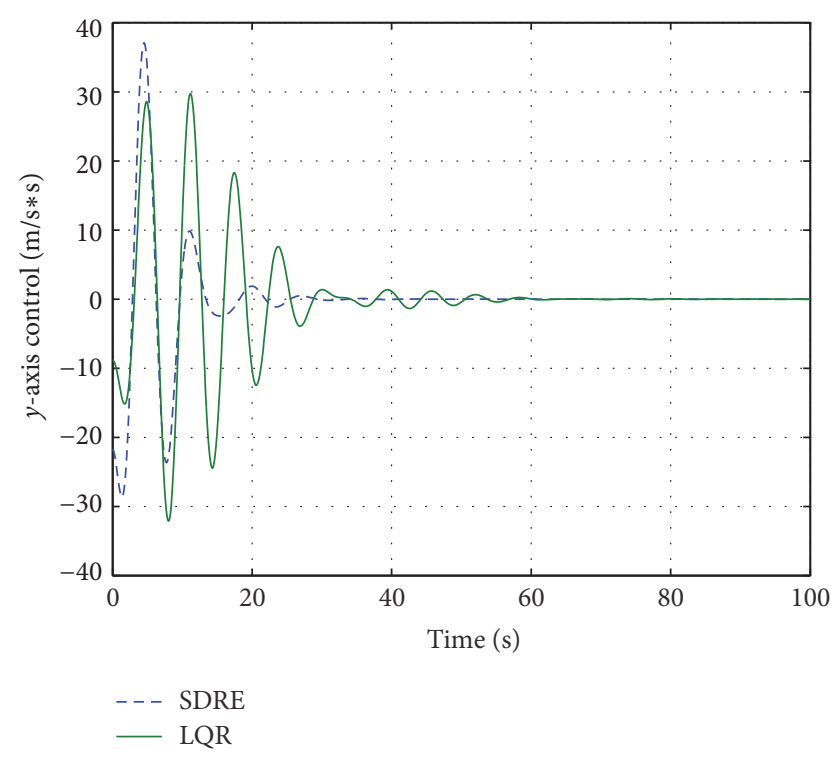

FIGURE 13: $Y$ axis control change curve.

method, the value of $R$ remains unchanged and the value of $Q$ increases, the steady-state time will become longer; the value of $Q$ remains unchanged and the value of $R$ increases, the overshooting volume of each state will increase. In the SDRE control method, when $Q(x)$ and $R(x)$ are different, the overshooting volume of each state and the time to reach the steady state are also different. But at the same level of weight matrix, the overshooting volume and steady-state time of SDRE control method are shorter than LQR control method.

In this paper, the nonlinear dynamic model of formation flying is established and the SDRE formation keeping controller is designed. Afterwards, simulation is carried out by using the related parameters of the sun-earth libration points. The simulation results are compared with LQR control method. In conclusion, SDRE control method is a control 


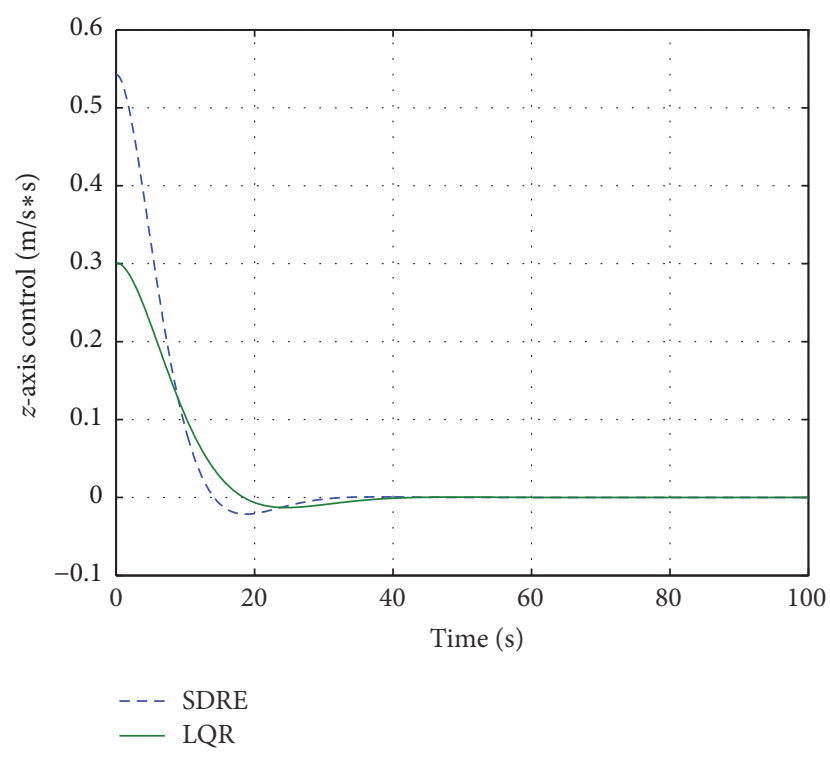

FIgURE 14: $Z$ axis control change curve.

method with high efficiency, low energy consumption on formation keeping for the sun-earth libration points and the theory can provide some reference value for practical use.

\section{Competing Interests}

The authors declare that there is no conflict of interests regarding the publication of this paper.

\section{References}

[1] M. Landgraf, F. Renk, and B. De Vogeleer, "Mission design and analysis of European astrophysics missions orbiting libration points," Acta Astronautica, vol. 84, pp. 49-55, 2013.

[2] Y. Lian, G. Gómez, J. J. Masdemont, and G. Tang, "tationkeeping of real Earth-Moon libration point orbits using discrete-time sliding mode contr," Communications in Nonlinear Science and Numerical Simulation, vol. 19, no. 10, pp. 37923807, 2014.

[3] J. V. Llop, "Autonomous optical navigation for orbits around Earth-Moon collinear libration points," Acta Astronautica, vol. 86, pp. 119-125, 2013.

[4] A. Barua and K. Khorasani, "Hierarchical fault diagnosis and health monitoring in satellites formation flight," IEEE Transactions on Systems, Man and Cybernetics Part C: Applications and Reviews, vol. 41, no. 2, pp. 223-239, 2011.

[5] H. H. Choi, "SDRE-based near optimal nonlinear controller design for unified chaotic systems," Nonlinear Dynamics, vol. 70, no. 3, pp. 2063-2070, 2012.

[6] M. Massari and M. Zamaro, "Application of SDRE technique to orbital and attitude control of spacecraft formation flying," Acta Astronautica, vol. 94, no. 1, pp. 409-420, 2014.

[7] Y.-W. Liang and L.-G. Lin, "Analysis of SDC matrices for successfully implementing the SDRE scheme," Automatica, vol. 49, no. 10, pp. 3120-3124, 2013.

[8] H. B. Duan and C. H. Sun, "Pendulum-like oscillation controller for micro aerial vehicle with ducted fan based on LQR and PSO,"
Science China Technological Sciences, vol. 56, no. 2, pp. 423-429, 2013.

[9] M. H. Korayem, A. Zehfroosh, H. Tourajizadeh, and S. Manteghi, "Optimal motion planning of non-linear dynamic systems in the presence of obstacles and moving boundaries using SDRE: application on cable-suspended robot," Nonlinear Dynamics, vol. 76, no. 2, pp. 1423-1441, 2014. 

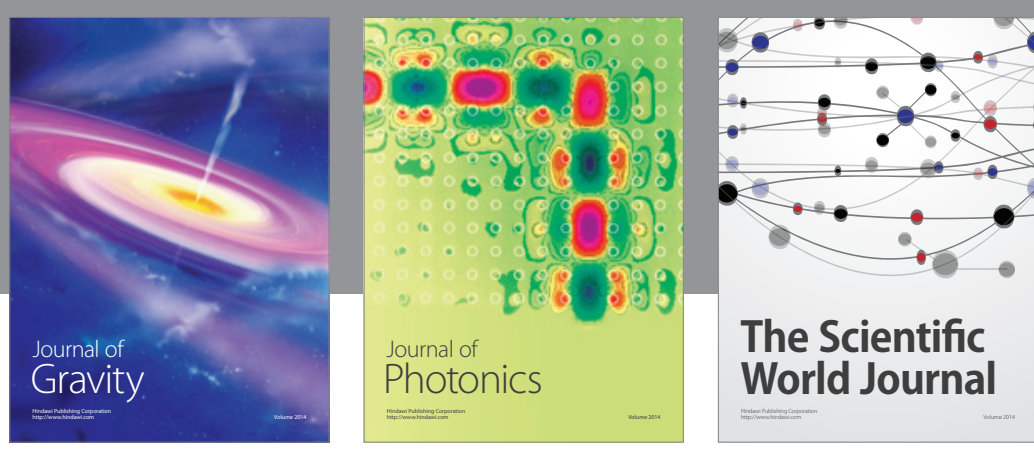

The Scientific World Journal
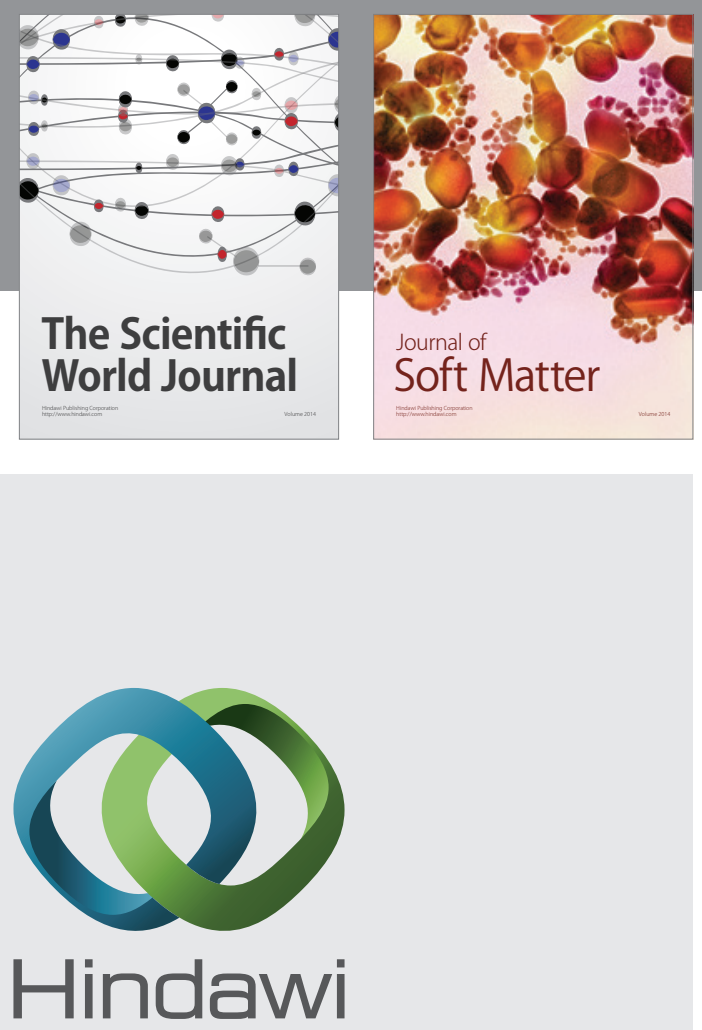

Submit your manuscripts at

https://www.hindawi.com
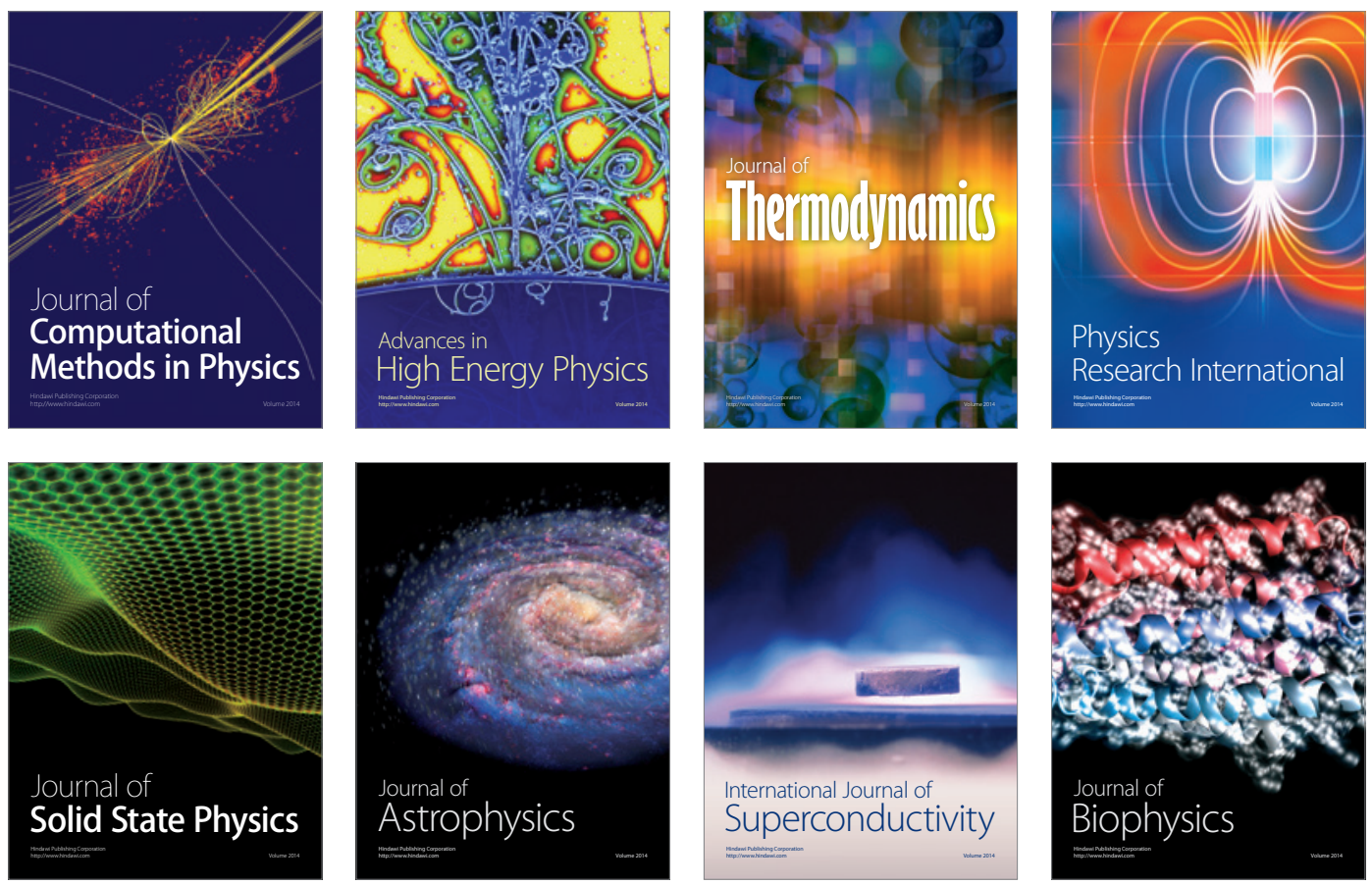
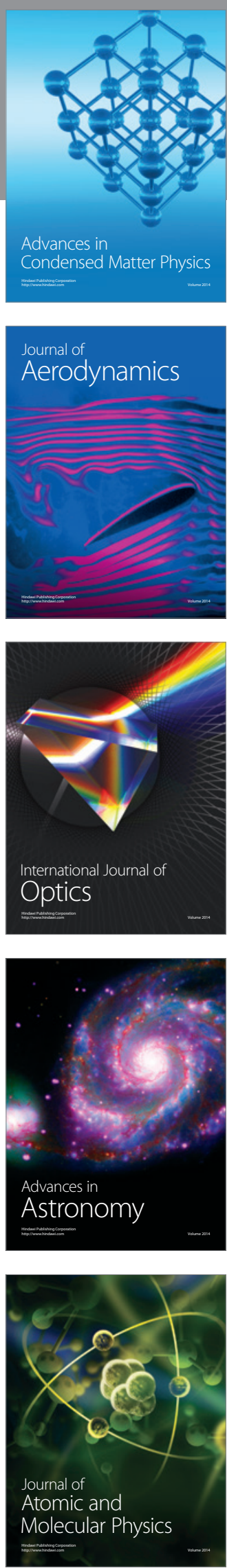